\title{
Stability analysis for a class of implicit fractional differential equations involving Atangana-Baleanu fractional derivative
}

\author{
Asma', Sana Shabbir ${ }^{1}$, Kamal Shah ${ }^{2,3}$ and Thabet Abdeljawad ${ }^{3,4,5^{*}}$ (D)
}

\section{"Correspondence:}

tabdeljawad@psu.edu.sa

${ }^{3}$ Department of Mathematics and

General Sciences, Prince Sultan

University, Riyadh, Saudi Arabia

${ }^{4}$ Department of Medical Research,

China Medical University, Taichung, 40402, Taiwan

Full list of author information is available at the end of the article

\begin{abstract}
Some fundamental conditions and hypotheses are established to ensure the existence, uniqueness, and stability to a class of implicit boundary value problems (BVPs) with Atangana-Baleanu-Caputo type derivative and integral. The required results are established by utilizing the Banach contraction mapping principle and fixed point theorem of Krasnoselskii. In addition, various types of stability results including Hyers-Ulam, generalized Hyers-Ulam, Hyers-Ulam-Rassias, and generalized Hyers-Ulam-Rassias stability are formulated for the problem under consideration. Pertinent examples are given to justify the results we obtain.
\end{abstract}

MSC: Primary 26A33; secondary 34A08; 93A30

Keywords: Atangana-Baleanu-Caputo derivative; Stability results; Fixed point theorems

\section{Introduction}

Our world is a combination of scientific phenomena. Mathematics is the mother of support to all sciences. Our many real life problems and many natural phenomena depend on or are governed by mathematical laws. Certain real life problems can be modeled and deeply studied by using calculus methods of integration and derivative. However, many problems can be studied more accurately by using fractional calculus methods. That is why fractional differential equations (FDEs) have become a major concern for many wellrenowned researchers and scientists. A fractional derivative of arbitrary order can be real or even a complex number as well. The credit for the first thought about fractional derivative goes to L. Hopital and Leibnitz in 1695 [1]. After this initial step, the researchers in this area kept on flourishing because of its numerous applications in physics, economics, engineering, and biological sciences.

In the last few years, FDEs got more attention. Many researchers also examined the solutions of fractional models for stability analysis [2]. Some researchers also worked on COVID-19 model with the help of fractional calculus [3, 4]. On the other hand, fixed point theory helps much in the development of the qualitative and computational concepts of FDEs and their related dynamic equations [5-11]. But still no fixed definition

(c) The Author(s) 2021. This article is licensed under a Creative Commons Attribution 4.0 International License, which permits use sharing, adaptation, distribution and reproduction in any medium or format, as long as you give appropriate credit to the original author(s) and the source, provide a link to the Creative Commons licence, and indicate if changes were made. The images or other third party material in this article are included in the article's Creative Commons licence, unless indicated otherwise in a credit line to the material. If material is not included in the article's Creative Commons licence and your intended use is not permitted by statutory regulation or exceeds the permitted use, you will need to obtain permission directly from the copyright holder. To view a copy of this licence, visit http://creativecommons.org/licenses/by/4.0/. 
of fractional operators has been defined. Researchers have worked on it rigorously and given many definitions with singular or nonsingular kernels. A lot of work has been done using Riemann-Liouville and Caputo fractional derivative and integration. After this Caputo and Fabrizio worked together and gave a new definition named Caputo-Fabrizio derivative. This derivative has a nonsingular kernel. After Caputo, Fabrizio, Atangana, and Baleanu gave another definition known as $\mathcal{A B}$ derivative. The $\mathcal{A B}$ derivative involves a nonlocal and nonsingular kernel [12]. The $\mathcal{A B}$ derivative, due to its Mittag-Leffler type kernel, has produced more interesting results as compared to Caputo-Fabrizio derivative whose exponential kernel can be splitter and hence affects the delay action. The new formulation of Caputo derivative by Atangana and Baleanu is known as Atangana-BaleanuCaputo $(\mathcal{A B C})$ and Riemann-Liouville $(\mathcal{A B R})$ derivatives [13]. Recently a significant contribution has been made by various authors and the field of FDEs (for details, see $[11,14-$ 17]).

Pantograph type FDEs play an important role in physics and applied mathematics. That's why these pantograph type delay FDEs have been deeply studied by many researchers. Jarad and his coauthors studied FDEs with Atangana-Baleanu-Caputo $(\mathcal{A B C})$ derivative [18] as follows:

$$
\left\{\begin{array}{l}
{ }_{\mathrm{a}}^{\mathcal{A B C}} \mathcal{D}_{\mathrm{t}}^{\alpha} \mathrm{u}(\mathrm{t})=\mathrm{g}(\mathrm{t}, \mathrm{u}(\mathrm{t})), \quad 0<\alpha \leq 1, \mathrm{t} \in[\mathrm{a}, \mathrm{b}] \\
\mathrm{u}(\mathrm{a})=\mathrm{u}_{\mathrm{a}}
\end{array}\right.
$$

Abdo and his coauthors worked on the following nonlinear pantograph FDEs $[13,19]$ :

$$
\left\{\begin{array}{l}
{ }_{\mathrm{a}}^{\mathcal{A B C}} \mathcal{D}_{\mathrm{t}}^{\alpha} \mathrm{u}(\mathrm{t})=\mathrm{g}(\mathrm{t}, \mathrm{u}(\mathrm{t}), \mathrm{u}(\gamma \mathrm{t})), \quad 0<\alpha \leq 1, \mathrm{t} \in[\mathrm{a}, \mathrm{b}] \\
\mathrm{u}(\mathrm{a})=\Sigma_{k=1}^{m} c_{k} \mathrm{u}\left(\mathrm{t}_{k}\right), \quad \mathrm{t}_{k} \in(\mathrm{a}, \mathrm{b}),
\end{array}\right.
$$

where $0<\alpha \leq 1$ is the order of derivative ${ }_{\mathrm{a}}{ }^{\mathcal{A B C}} \mathcal{D}_{\mathrm{t}}^{\alpha}$, $\mathrm{g}:[\mathrm{a}, \mathrm{b}] \times \mathcal{R} \times \mathcal{R} \rightarrow \mathcal{R}$ is a continuous function, and $\gamma \in(0,1]$.

In the last few years the area devoted to investigating initial and BVPs under $\mathcal{A B C}$ fractional order derivative has been developed very well. Here, we remark that the area devoted to studying biological models under the said derivative has been investigated very well. Numerical interpretations for various kinds of FDEs under the aforesaid derivative have been studied very well in the last few years [20]. Inspired by the research work as mentioned above, we intend to work on a implicit BVP involving $(\mathcal{A B C})$ derivative of the form:

$$
\left\{\begin{array}{l}
{ }_{0}^{\mathcal{A B C}} \mathcal{D}_{\mathrm{t}}^{\alpha} \mathrm{u}(\mathrm{t})=\mathrm{g}\left(\mathrm{t}, \mathrm{u}(\mathrm{t}),{ }_{0}^{\mathcal{A B C}} \mathcal{D}_{\mathrm{t}}^{\alpha} \mathrm{u}(\mathrm{t})\right), \quad 1<\alpha \leq 2, \mathrm{t} \in \mathcal{J}=[0, \mathrm{~b}] \\
\mathrm{u}(0)=\mathrm{u}_{0}, \quad \mathrm{u}(\mathrm{b})=\mathrm{u}_{1}
\end{array}\right.
$$

where ${ }_{0}^{\mathcal{A B C}} \mathcal{D}_{\mathrm{t}}^{\alpha}$ represents the $\mathcal{A B C}$ derivative of order $1<\alpha \leq 2$ and $\mathrm{g}: \mathcal{J} \times \mathcal{R} \times \mathcal{R} \longrightarrow \mathcal{R}$ is a continuous function.

We compute results for implicit FDEs with boundary conditions. Most of our derivations are made using theorems of significant importance such as Krasnoselskii, Arzelá-Ascoli and Banach fixed point theorems. Also we derive various results for Ulam-Hyers type stability analysis of our problem as the said stability has been investigated in the last few 
years very well in respect of FDEs. Also some researchers have investigated various results of the concerned stability in nonlinear analysis (see [21-25]).

\section{Fundamental results}

Here, we recollect some basic definitions of fractional calculus which are needed throughout this work. We derive our main results by using these fundamental results. Suppose that $\mathbb{X}=\mathcal{C}[\mathcal{J}, \mathcal{R}]$ is a Banach space and the norm under consideration is $\|\mathrm{u}\|=\max _{\mathrm{t} \in \mathcal{J}}|\mathrm{u}(\mathrm{t})|$.

Definition 1 ([26, 27] $\mathcal{A B C}$ fractional derivative) Let $\mathrm{u} \in \mathrm{H}^{1}(c, d), c<d$, and $\alpha \in(0,1)$. The $\mathcal{A B C}$ fractional derivative for function $\mathrm{u}$ of order $\alpha$ is defined as

$$
{ }_{0}^{A \mathcal{B C}} \mathcal{D}_{\mathrm{t}}^{\alpha} \mathrm{u}(\mathrm{t})=\frac{\mathbb{M}(\alpha)}{1-\alpha} \int_{0}^{\mathrm{t}} \mathrm{u}^{\prime}(\xi) \mathrm{E}_{\alpha}\left(\frac{-\alpha(\mathrm{t}-\xi)^{\alpha}}{1-\alpha}\right) d \xi
$$

We use this definition throughout the paper. Further, the mentioned operator in the Riemann-Liouville sense is defined as

$$
{ }_{0}^{\mathcal{A B R}} \mathcal{D}_{\mathrm{t}}^{\alpha} \mathrm{u}(\mathrm{t})=\frac{\mathbb{M}(\alpha)}{1-\alpha} \frac{d}{d \mathrm{t}} \int_{0}^{\mathrm{t}} \mathrm{u}(\xi) \mathrm{E}_{\alpha}\left(\frac{-\alpha(\mathrm{t}-\xi)^{\alpha}}{1-\alpha}\right) d \xi
$$

In the above equations $\mathbb{M}(\alpha)>0$ is a normalization function. Here, $\mathbb{M}(0)=\mathbb{M}(1)=1$ and $\mathrm{E}_{\alpha}$ represents the well-known Mittag-Leffler function.

Definition $2([26,27] \mathcal{A B}$ fractional integral) Let u be a function, then the $\mathcal{A B}$ fractional integral of order $\alpha \in(0,1)$ is defined by

$$
{ }_{0}^{\mathcal{A}} \mathcal{I}_{\mathrm{t}}^{\alpha} \mathrm{u}(\mathrm{t})=\frac{1-\alpha}{\mathbb{M}(\alpha)} \mathrm{u}(\mathrm{t})+\frac{\alpha}{\mathbb{M}(\alpha) \Gamma(\alpha)} \int_{0}^{\mathrm{t}} \mathrm{u}(\xi)(\mathrm{t}-\xi)^{\alpha-1} d \xi
$$

Definition 3 ([28]) Let $\mathrm{u}$ be a function such that $\mathrm{u}^{(n)} \in \mathrm{H}^{1}(c, d)$ and $n<\alpha \leq n+1, n=$ $0,1, \ldots$ Then the $\mathcal{A B C}$ derivative satisfies the following formula:

$$
{ }_{0}^{\mathcal{A B C}} \mathcal{D}_{\mathrm{t}}^{\alpha} \mathrm{u}(\mathrm{t})={ }_{0}^{\mathcal{A B C}} \mathcal{D}_{\mathrm{t}}^{\beta} \mathrm{u}^{(n)}(\mathrm{t})
$$

where $\beta=\alpha-n$.

Lemma 1 ([28]) For $\alpha \in(n, n+1], n=0,1,2, \ldots$, the following outcome holds for the FDEs:

$$
{ }_{0}^{\mathcal{A B}} \mathcal{I}_{\mathrm{t} 0}^{\alpha \cdot \mathcal{A B C}} \mathcal{D}^{\alpha}{ }_{\mathrm{t}} \mathrm{u}(\mathrm{t})=\mathrm{u}(\mathrm{t})+\mathfrak{c}_{0}+\mathfrak{c}_{1} \mathrm{t}+\mathfrak{c}_{2} \mathrm{t}^{2}+\cdots+\mathfrak{c}_{n} \mathrm{t}^{n}
$$

for an arbitrary constant $\mathfrak{c}_{i}$ with $i=0,1,2, \ldots, n$.

Theorem $1([29,30])$ Let $\mathcal{W}$ be a nonempty, convex, and closed subset of $\mathbb{X}$. Consider two operators $\mathcal{H}, \mathcal{B}$ such that

(1) $\mathcal{H}\left(w_{1}\right)+\mathcal{B}\left(w_{2}\right) \in \mathcal{W}$ for all $w_{1}, w_{2} \in \mathcal{W}$,

(2) $\mathcal{H}$ is a contraction operator,

(3) $\mathcal{B}$ is continuous and compact,

then there exists at least one solution $w \in \mathbb{X}$, such that $\mathcal{H}(w)+\mathcal{B}(w)=w$. 


\section{Mathematical analysis of model problem}

The present section of our paper is reserved to examining the existence and uniqueness for the solution of our implicit FDEs by Krasnoselskii's fixed point theorem.

Lemma 2 Let $\varpi \in \mathbb{X}$, then our problem can be formulated as

$$
\left\{\begin{array}{l}
{ }_{0}^{\mathcal{B} C} \mathcal{D}_{\mathrm{t}}^{\alpha} \mathrm{u}(\mathrm{t})=\varpi(\mathrm{t}), \quad 1<\alpha \leq 2, \mathrm{t} \in \mathcal{J} \\
\mathrm{u}(0)=\mathrm{u}_{0}, \quad \mathrm{u}(\mathrm{b})=\mathrm{u}_{1} .
\end{array}\right.
$$

The solution of the above problem is given by

$$
\begin{aligned}
\mathrm{u}(\mathrm{t})= & \frac{\mathrm{tu}_{1}+\mathrm{u}_{0}(\mathrm{~b}-\mathrm{t})}{\mathrm{b}}-\frac{\mathrm{t}(2-\alpha)}{\mathrm{bM}(\alpha-1)} \int_{0}^{\mathrm{b}} \varpi(\xi) d \xi \\
& -\frac{\mathrm{t}(\alpha-1)}{\mathrm{bM}(\alpha-1) \Gamma(\alpha)} \int_{0}^{\mathrm{b}} \varpi(\xi)(\mathrm{b}-\xi)^{\alpha-1} d \xi \\
& +\frac{2-\alpha}{\mathbb{M}(\alpha-1)} \int_{0}^{\mathrm{t}} \varpi(\xi) d \xi+\frac{\alpha-1}{\mathbb{M}(\alpha-1) \Gamma(\alpha)} \int_{0}^{\mathrm{t}} \varpi(\xi)(\mathrm{t}-\xi)^{\alpha-1} d \xi
\end{aligned}
$$

Proof Consider ${ }_{0}^{\mathcal{A B C}} \mathcal{D}_{\mathrm{t}}^{\alpha} \mathrm{u}(\mathrm{t})=\varpi(\mathrm{t})$. Then, by applying the integral ${ }_{0}^{\mathcal{A B}} \mathcal{I}_{\mathrm{t}}^{\alpha}$ on both sides, we get

$$
\mathrm{u}(\mathrm{t})=\mathfrak{c}_{0}+\mathfrak{c}_{1} \mathrm{t}+\frac{2-\alpha}{\mathbb{M}(\alpha-1)} \int_{0}^{\mathrm{t}} \varpi(\xi) d \xi+\frac{\alpha-1}{\mathbb{M}(\alpha-1) \Gamma(\alpha)} \int_{0}^{\mathrm{t}} \varpi(\xi)(\mathrm{t}-\xi)^{\alpha-1} d \xi .
$$

Now, using the boundary condition $\mathrm{u}(0)=\mathrm{u}_{0}$, we get $\mathfrak{c}_{0}=\mathrm{u}_{0}$.

Now, for $\mathrm{u}(\mathrm{b})=\mathrm{u}_{1}$, we get

$$
\mathfrak{c}_{1}=\frac{\mathrm{u}_{1}-\mathrm{u}_{0}}{\mathrm{~b}}-\frac{2-\alpha}{\mathrm{bM}(\alpha-1)} \int_{0}^{\mathrm{b}} \varpi(\xi) d \xi-\frac{\alpha-1}{\mathrm{bM}(\alpha-1) \Gamma(\alpha)} \int_{0}^{\mathrm{b}} \varpi(\xi)(\mathrm{b}-\xi)^{\alpha-1} d \xi .
$$

Substituting the values of $\mathfrak{c}_{0}$ and $\mathfrak{c}_{1}$ in equation (8), we get

$$
\begin{aligned}
\mathrm{u}(\mathrm{t})= & \frac{\mathrm{tu}_{1}+\mathrm{u}_{0}(\mathrm{~b}-\mathrm{t})}{\mathrm{b}}-\frac{\mathrm{t}(2-\alpha)}{\mathrm{bM}(\alpha-1)} \int_{0}^{\mathrm{b}} \varpi(\xi) d \xi \\
& -\frac{\mathrm{t}(\alpha-1)}{\mathrm{bM}(\alpha-1) \Gamma(\alpha)} \int_{0}^{\mathrm{b}} \varpi(\xi)(\mathrm{b}-\xi)^{\alpha-1} d \xi \\
& +\frac{2-\alpha}{\mathbb{M}(\alpha-1)} \int_{0}^{\mathrm{t}} \varpi(\xi) d \xi+\frac{\alpha-1}{\mathbb{M}(\alpha-1) \Gamma(\alpha)} \int_{0}^{\mathrm{t}} \varpi(\xi)(\mathrm{t}-\xi)^{\alpha-1} d \xi .
\end{aligned}
$$

Corollary 1 In view of Lemma 2, our problem (3) is equivalent to the following integral equation:

$$
\begin{aligned}
\mathrm{u}(\mathrm{t})= & \frac{\mathrm{tu}_{1}+\mathrm{u}_{0}(\mathrm{~b}-\mathrm{t})}{\mathrm{b}}-\frac{\mathrm{t}(2-\alpha)}{\mathrm{bM}(\alpha-1)} \int_{0}^{\mathrm{b}} \mathrm{g}\left(\xi, \mathrm{u}(\xi),{ }_{0}^{\mathcal{A}}{ }^{\mathcal{B C}} \mathcal{D}_{\xi}^{\alpha} \mathrm{u}(\xi)\right) d \xi \\
& -\frac{\mathrm{t}(\alpha-1)}{\mathrm{bM}(\alpha-1) \Gamma(\alpha)} \int_{0}^{\mathrm{b}} \mathrm{g}\left(\xi, \mathrm{u}(\xi),{ }_{0}^{\mathcal{A B C}} \mathcal{D}_{\xi}^{\alpha} \mathrm{u}(\xi)\right)(\mathrm{b}-\xi)^{\alpha-1} d \xi \\
& +\frac{2-\alpha}{\mathbb{M}(\alpha-1)} \int_{0}^{\mathrm{t}} \mathrm{g}\left(\xi, \mathrm{u}(\xi),{ }_{0}^{\mathcal{A B C}} \mathcal{D}_{\xi}^{\alpha} \mathrm{u}(\xi)\right) d \xi
\end{aligned}
$$




$$
+\frac{\alpha-1}{\mathrm{M}(\alpha-1) \Gamma(\alpha)} \int_{0}^{\mathrm{t}} \mathrm{g}\left(\xi, \mathrm{u}(\xi),{ }_{0}^{\mathcal{A} B \mathcal{C} C} \mathcal{D}_{\xi}^{\alpha} \mathrm{u}(\xi)\right)(\mathrm{t}-\xi)^{\alpha-1} d \xi
$$

For the proof of our main results, we employ the following hypotheses:

$\left(\mathrm{A}_{1}\right)$ There exists a constant $\mathcal{L}_{\mathrm{g}}>0$ such that, for any $\mathrm{u}, \overline{\mathrm{u}}, \mathrm{v}, \overline{\mathrm{v}} \in \mathbb{X}$, one has

$$
|g(t, u, v)-g(t, \bar{u}, \bar{v})| \leq \mathcal{L}_{g}(|u-\bar{u}|+|v-\bar{v}|) .
$$

$\left(\mathrm{A}_{2}\right)$ There exist constants $\mathbf{A}_{\mathrm{g}}, \mathbf{B}_{\mathrm{g}}, \mathbf{C}_{\mathrm{g}}>0$ such that

$$
|\mathrm{g}(\mathrm{t}, \mathrm{u}, \mathrm{v})| \leq \mathbf{A}_{\mathrm{g}}+\mathbf{B}_{\mathrm{g}}|\mathrm{u}|+\mathbf{C}_{\mathrm{g}}|\mathrm{v}|
$$

Theorem 2 Under assumption $\left(\mathrm{A}_{1}\right), B V P(3)$ has the unique solution if

$$
4\left(\mathcal{L}_{\mathrm{g}}+\frac{\mathcal{L}_{\mathrm{g}}^{2}}{1-\mathcal{L}_{\mathrm{g}}}\right) \frac{\mathrm{b} \Gamma(\alpha+1)+\mathrm{b}^{\alpha}}{\mathrm{M}(\alpha-1) \Gamma(\alpha+1)}<1 .
$$

Proof Let $\mathcal{H}: \mathbb{X} \rightarrow \mathbb{X}$ be defined by

$$
\begin{aligned}
\mathcal{H} \mathrm{u}(\mathrm{t})= & \frac{\mathrm{tu}_{1}+\mathrm{u}_{0}(\mathrm{~b}-\mathrm{t})}{\mathrm{b}}-\frac{\mathrm{t}(2-\alpha)}{\mathrm{bM}(\alpha-1)} \int_{0}^{\mathrm{b}} \mathrm{g}\left(\xi, \mathrm{u}(\xi),{ }_{0}^{\mathcal{A B C}} \mathcal{D}_{\xi}^{\alpha} \mathrm{u}(\xi)\right) d \xi \\
& -\frac{\mathrm{t}(\alpha-1)}{\mathrm{bM}(\alpha-1) \Gamma(\alpha)} \int_{0}^{\mathrm{b}} \mathrm{g}\left(\xi, \mathrm{u}(\xi),{ }_{0}^{\mathcal{A B C}} \mathcal{D}_{\xi}^{\alpha} \mathrm{u}(\xi)\right)(\mathrm{b}-\xi)^{\alpha-1} d \xi \\
& +\frac{2-\alpha}{\mathbb{M}(\alpha-1)} \int_{0}^{\mathrm{t}} \mathrm{g}\left(\xi, \mathrm{u}(\xi),{ }_{0}^{\mathcal{A} \mathcal{B C}} \mathcal{D}_{\xi}^{\alpha} \mathrm{u}(\xi)\right) d \xi \\
& +\frac{\alpha-1}{\mathbb{M}(\alpha-1) \Gamma(\alpha)} \int_{0}^{\mathrm{t}} \mathrm{g}\left(\xi, \mathrm{u}(\xi),{ }_{0}^{\mathcal{A}}{ }^{\mathcal{B} C} \mathcal{D}_{\xi}^{\alpha} \mathrm{u}(\xi)\right)(\mathrm{t}-\xi)^{\alpha-1} d \xi .
\end{aligned}
$$

To examine the uniqueness of the solution of the problem, firstly we show that $\mathcal{H}$ is a contraction operator. To this end, suppose $\mathrm{u}, \overline{\mathrm{u}} \in \mathbb{X}$, then

$$
\begin{aligned}
& \|\mathcal{H u}-\mathcal{H} \overline{\mathrm{u}}\| \\
& =\max _{\mathrm{t} \in \mathcal{J}} \mid \frac{\mathrm{t}(2-\alpha)}{\mathrm{bM}(\alpha-1)} \int_{0}^{\mathrm{b}}\left[\mathrm{g}\left(\xi, \mathrm{u}(\xi),{ }_{0}^{\mathcal{A B C}} \mathcal{D}_{\xi}^{\alpha} \mathrm{u}(\xi)\right)-\mathrm{g}\left(\xi, \overline{\mathrm{u}}(\xi),{ }_{0}^{\mathcal{A B C}} \mathcal{D}_{\xi}^{\alpha} \overline{\mathrm{u}}(\xi)\right)\right] d \xi \\
& +\frac{\mathrm{t}(\alpha-1)}{\mathrm{bM}(\alpha-1) \Gamma(\alpha)} \int_{0}^{\mathrm{b}}\left[\mathrm{g}\left(\xi, \mathrm{u}(\xi),{ }_{0}^{\mathcal{A B C}} \mathcal{D}_{\xi}^{\alpha} \mathrm{u}(\xi)\right)-\mathrm{g}\left(\xi, \overline{\mathrm{u}}(\xi),{ }_{0}^{\mathcal{A B C}} \mathcal{D}_{\xi}^{\alpha} \overline{\mathrm{u}}(\xi)\right)\right] \\
& \times(\mathrm{b}-\xi)^{\alpha-1} d \xi \\
& +\frac{2-\alpha}{\mathbb{M}(\alpha-1)} \int_{0}^{\mathrm{b}}\left[\mathrm{g}\left(\xi, \mathrm{u}(\xi),{ }_{0}^{\mathcal{A B C}} \mathcal{D}_{\xi}^{\alpha} \mathrm{u}(\xi)\right)-\mathrm{g}\left(\xi, \overline{\mathrm{u}}(\xi),{ }_{0}^{\mathcal{A B C}} \mathcal{D}_{\xi}^{\alpha} \overline{\mathrm{u}}(\xi)\right)\right] d \xi \\
& +\frac{\alpha-1}{\mathrm{M}(\alpha-1) \Gamma(\alpha)} \int_{0}^{\mathrm{b}}\left[\mathrm{g}\left(\xi, \mathrm{u}(\xi),{ }_{0}^{\mathcal{A B C}} \mathcal{D}_{\xi}^{\alpha} \mathrm{u}(\xi)\right)-\mathrm{g}\left(\xi, \overline{\mathrm{u}}(\xi),{ }_{0}^{\mathcal{A B C}} \mathcal{D}_{\xi}^{\alpha} \overline{\mathrm{u}}(\xi)\right)\right] \\
& \times(\mathrm{t}-\xi)^{\alpha-1} d \xi \\
& \leq \max _{\mathrm{t} \in \mathcal{J}}\left[\frac{|\mathrm{t}|(2-\alpha)}{\mathrm{bM}(\alpha-1)} \int_{0}^{\mathrm{b}} \mathcal{L}_{\mathrm{g}}\left(|\mathrm{u}(\xi)-\overline{\mathrm{u}}(\xi)|+\left.\right|_{0} ^{\mathcal{A B C}} \mathcal{D}_{\xi}^{\alpha} \mathrm{u}(\xi)-{ }_{0}^{\mathcal{A B C}} \mathcal{D}_{\xi}^{\alpha} \overline{\mathrm{u}}(\xi) \mid\right) d \xi\right.
\end{aligned}
$$




$$
\begin{aligned}
& +\frac{|\mathrm{t}|(\alpha-1)}{\mathrm{b} \mathbb{M}(\alpha-1) \Gamma(\alpha)} \int_{0}^{\mathrm{b}} \mathcal{L}_{\mathrm{g}}\left(|\mathrm{u}(\xi)-\overline{\mathrm{u}}(\xi)|+\left|{ }_{0}^{\mathcal{A B C}} \mathcal{D}_{\xi}^{\alpha} \mathrm{u}(\xi)-{ }_{0}^{\mathcal{A B C}} \mathcal{D}_{\xi}^{\alpha} \overline{\mathrm{u}}(\xi)\right|\right) \\
& \times(\mathrm{b}-\xi)^{\alpha-1} d \xi \\
& +\frac{2-\alpha}{\mathbb{M}(\alpha-1)} \int_{0}^{\mathrm{b}} \mathcal{L}_{\mathrm{g}}\left(|\mathrm{u}(\xi)-\overline{\mathrm{u}}(\xi)|+\left|{ }_{0}^{\mathcal{A B C}} \mathcal{D}_{\xi}^{\alpha} \mathrm{u}(\xi)-{ }_{0}^{\mathcal{A B C}} \mathcal{D}_{\xi}^{\alpha} \overline{\mathrm{u}}(\xi)\right|\right) d \xi \\
& \left.+\frac{\alpha-1}{\mathbb{M}(\alpha-1) \Gamma(\alpha)} \int_{0}^{\mathrm{b}} \mathcal{L}_{\mathrm{g}}\left(|\mathrm{u}(\xi)-\overline{\mathrm{u}}(\xi)|+\left|{ }_{0}^{\mathcal{A B C}} \mathcal{D}_{\xi}^{\alpha} \mathrm{u}(\xi)-{ }_{0}^{\mathcal{A B C}} \mathcal{D}_{\xi}^{\alpha} \overline{\mathrm{u}}(\xi)\right|\right) d \xi\right] .
\end{aligned}
$$

Now, we have

$$
\begin{aligned}
& \quad\left|{ }_{0}^{\mathcal{A B C}} \mathcal{D}_{\xi}^{\alpha} \mathrm{u}(\mathrm{t})-{ }_{0}^{\mathcal{A}}{ }^{\mathcal{B C}} \mathcal{D}_{\xi}^{\alpha} \overline{\mathrm{u}}(\mathrm{t})\right| \\
& \quad=\left|\mathrm{g}\left(\mathrm{t}, \mathrm{u}(\mathrm{t}),{ }_{0}^{\mathcal{A} B C} \mathcal{D}_{\xi}^{\alpha} \mathrm{u}(\mathrm{t})\right)-\mathrm{g}\left(\mathrm{t}, \overline{\mathrm{u}}(\mathrm{t}),{ }_{0}^{\mathcal{A B C}} \mathcal{D}_{\xi}^{\alpha} \overline{\mathrm{u}}(\mathrm{t})\right)\right| \\
& \quad \leq \mathcal{L}_{\mathrm{g}}\left(|\mathrm{u}(\mathrm{t})-\overline{\mathrm{u}}(\mathrm{t})|+\left|{ }_{0}^{\mathcal{A B C}} \mathcal{D}_{\xi}^{\alpha} \mathrm{u}(\mathrm{t})-{ }_{0}^{\mathcal{A B C}} \mathcal{D}_{\xi}^{\alpha} \overline{\mathrm{u}}(\mathrm{t})\right|\right) .
\end{aligned}
$$

This implies

$$
\left|{ }_{0}^{\mathcal{A B C}} \mathcal{D}_{\xi}^{\alpha} \mathrm{u}(\xi)-{ }_{0}^{\mathcal{A B C}} \mathcal{D}_{\xi}^{\alpha} \overline{\mathrm{u}}(\mathrm{t})\right| \leq\left(\frac{\mathcal{L}_{\mathrm{g}}}{1-\mathcal{L}_{\mathrm{g}}}\right)|\mathrm{u}(\mathrm{t})-\overline{\mathrm{u}}(\mathrm{t})| .
$$

Using equation (11) in equation (10), we get

$$
\|\mathcal{H} \mathrm{u}-\mathcal{H} \overline{\mathrm{u}}\| \leq 4\left(\mathcal{L}_{\mathrm{g}}+\frac{\mathcal{L}_{\mathrm{g}}^{2}}{1-\mathcal{L}_{\mathrm{g}}}\right) \frac{\mathrm{b} \Gamma(\alpha+1)+\mathrm{b}^{\alpha}}{\mathrm{M}(\alpha-1) \Gamma(\alpha+1)}\|\mathrm{u}-\overline{\mathrm{u}}\| .
$$

In view of equation (9), our BVP (3) has the unique solution. Hence $\mathcal{H}$ is a contraction operator. Hence $\mathcal{H}$ is defined under consideration of Krasnoselskii's fixed point theorem as follows:

$$
\mathcal{H} \mathrm{u}(\mathrm{t})=\mathcal{F} \mathrm{u}(\mathrm{t})+\mathcal{N} \mathrm{u}(\mathrm{t})
$$

where

$$
\begin{aligned}
\mathcal{F} \mathrm{u}(\mathrm{t})= & \frac{\mathrm{tu}_{1}+\mathrm{u}_{0}(\mathrm{~b}-\mathrm{t})}{\mathrm{b}}-\frac{\mathrm{t}(2-\alpha)}{\mathrm{bM}(\alpha-1)} \int_{0}^{\mathrm{b}} \mathrm{g}\left(\xi, \mathrm{u}(\xi),{ }_{0}^{\mathcal{A} B C} \mathcal{D}_{\xi}^{\alpha} \mathrm{u}(\xi)\right) d \xi \\
& -\frac{\mathrm{t}(\alpha-1)}{\mathrm{bM}(\alpha-1) \Gamma(\alpha)} \int_{0}^{\mathrm{b}} \mathrm{g}\left(\xi, \mathrm{u}(\xi),{ }_{0}^{\mathcal{A} B C} \mathcal{D}_{\xi}^{\alpha} \mathrm{u}(\xi)\right)(\mathrm{b}-\xi)^{\alpha-1} d \xi \\
\mathcal{N} \mathrm{u}(\mathrm{t})= & +\frac{2-\alpha}{\mathbb{M}(\alpha-1)} \int_{0}^{\mathrm{t}} \mathrm{g}\left(\xi, \mathrm{u}(\xi),{ }_{0}^{\mathcal{A B C}} \mathcal{D}_{\xi}^{\alpha} \mathrm{u}(\xi)\right) d \xi \\
& +\frac{\alpha-1}{\mathbb{M}(\alpha-1) \Gamma(\alpha)} \int_{0}^{\mathrm{t}} \mathrm{g}\left(\xi, \mathrm{u}(\xi),{ }_{0}^{\mathcal{A B C}} \mathcal{D}_{\xi}^{\alpha} \mathrm{u}(\xi)\right)(\mathrm{t}-\xi)^{\alpha-1} d \xi
\end{aligned}
$$

Theorem 3 Under the contemplation of assumptions $\left(\mathrm{A}_{1}\right)$ and $\left(\mathrm{A}_{2}\right)$, our BVP has at least one solution with the condition

$$
2\left(\mathcal{L}_{\mathrm{g}}+\frac{\mathcal{L}_{\mathrm{g}}^{2}}{1-\mathcal{L}_{\mathrm{g}}}\right) \frac{\mathrm{b} \Gamma(\alpha+1)+\mathrm{b}^{\alpha}}{\mathrm{M}(\alpha-1) \Gamma(\alpha+1)}<1 .
$$


Proof Let $\mathcal{W}=\{\mathrm{u} \in \mathbb{X}:\|\mathrm{u}\| \leq \mathrm{r}\}$ be a closed bounded set and $\mathrm{u}, \overline{\mathrm{u}} \in \mathcal{W}$. Now

$$
\begin{aligned}
\| \mathcal{F} \mathrm{u}- & \mathcal{F} \overline{\mathrm{u}} \| \\
= & \max _{\mathrm{t} \in \mathcal{J}}|\mathcal{F} \mathrm{u}(\mathrm{t})-\mathcal{F} \overline{\mathrm{u}}(\mathrm{t})| \\
= & \max _{\mathrm{t} \in \mathcal{J}} \frac{|\mathrm{t}(2-\alpha)|}{\mathrm{bM}(\alpha-1)} \int_{0}^{\mathrm{b}}\left|\mathrm{g}\left(\xi, \mathrm{u}(\xi),{ }_{0}^{\mathcal{A B C}} \mathcal{D}_{\xi}^{\alpha} \mathrm{u}(\xi)\right)-\mathrm{g}\left(\xi, \overline{\mathrm{u}}(\xi),{ }_{0}^{\mathcal{A B C}} \mathcal{D}_{\xi}^{\alpha} \overline{\mathrm{u}}(\xi)\right)\right| d \xi \\
& +\max _{\mathrm{t} \in \mathcal{J}} \frac{|\mathrm{t}|(\alpha-1)}{\mathrm{bM}(\alpha-1) \Gamma(\alpha)} \int_{0}^{\mathrm{b}}\left|\mathrm{g}\left(\xi, \mathrm{u}(\xi),{ }_{0}^{\mathcal{A B C}} \mathcal{D}_{\xi}^{\alpha} \mathrm{u}(\xi)\right)-\mathrm{g}\left(\xi, \overline{\mathrm{u}}(\xi),{ }_{0}^{\mathcal{A B C}} \mathcal{D}_{\xi}^{\alpha} \overline{\mathrm{u}}(\xi)\right)\right| \\
& \times(\mathrm{b}-\xi)^{\alpha-1} d \xi \\
\leq & \max _{\mathrm{t} \in \mathcal{J}} \frac{|\mathrm{t}|(2-\alpha)}{\mathrm{bM}(\alpha-1)} \int_{0}^{\mathrm{b}} \mathcal{L}_{\mathrm{g}}\left(|\mathrm{u}(\xi)-\overline{\mathrm{u}}(\xi)|+\left|{ }_{0}^{\mathcal{A B C}} \mathcal{D}_{\xi}^{\alpha} \mathrm{u}(\xi)-{ }_{0}^{\mathcal{A B C}} \mathcal{D}_{\xi}^{\alpha} \overline{\mathrm{u}}(\xi)\right|\right) d \xi \\
& +\max _{\mathrm{t} \in \mathcal{J}} \frac{|\mathrm{t}|(\alpha-1)}{\mathrm{bM}(\alpha-1) \Gamma(\alpha)} \int_{0}^{\mathrm{b}} \mathcal{L}_{\mathrm{g}}\left(|\mathrm{u}(\xi)-\overline{\mathrm{u}}(\xi)|+\left|{ }_{0}^{\mathcal{A B C}} \mathcal{D}_{\xi}^{\alpha} \mathrm{u}(\xi)-{ }_{0}^{\mathcal{A B C}} \mathcal{D}_{\xi}^{\alpha} \overline{\mathrm{u}}(\xi)\right|\right) \\
& \times(\mathrm{b}-\xi)^{\alpha-1} d \xi \\
\leq & 2\left(\mathcal{L}_{\mathrm{g}}+\frac{\mathcal{L}_{\mathrm{g}}^{2}}{1-\mathcal{L}_{\mathrm{g}}}\right) \frac{\mathrm{b} \Gamma(\alpha+1)+\mathrm{b}^{\alpha}}{\mathbb{M}(\alpha-1) \Gamma(\alpha+1)}\|\mathrm{u}-\overline{\mathrm{u}}\| .
\end{aligned}
$$

In view of inequality (12), $\mathcal{F}$ is a contraction mapping. The next step is to prove the continuity and compactness for $\mathcal{N}$. We show that our operator $\mathcal{N}$ is bounded. To examine the equicontinuity for the operator, one has

$$
\begin{aligned}
\|\mathcal{N} \mathrm{u}\|= & \max _{\mathrm{t} \in \mathcal{J}}|\mathcal{N} \mathrm{u}(\mathrm{t})| \\
= & \max _{\mathrm{t} \in \mathcal{J}} \mid \frac{2-\alpha}{\mathbb{M}(\alpha-1)} \int_{0}^{\mathrm{t}} \mathrm{g}\left(\xi, \mathrm{u}(\xi),{ }_{0}^{\mathcal{A B C}} \mathcal{D}_{\xi}^{\alpha} \mathrm{u}(\xi)\right) d \xi \\
& +\frac{\alpha-1}{\mathbb{M}(\alpha-1) \Gamma(\alpha)} \int_{0}^{\mathrm{t}} \mathrm{g}\left(\xi, \mathrm{u}(\xi),{ }_{0}^{\mathcal{A B C}} \mathcal{D}_{\xi}^{\alpha} \mathrm{u}(\xi)\right)(\mathrm{t}-\xi)^{\alpha-1} d \xi \mid \\
\leq & \frac{2-\alpha}{\mathbb{M}(\alpha-1)} \int_{0}^{\mathrm{t}}\left[\mathbf{A}_{\mathrm{g}}+\mathbf{B}_{\mathrm{g}}|\mathrm{u}(\xi)|+\mathbf{C}_{\mathrm{g}}\left|{ }_{0}^{\mathcal{A B C}} \mathcal{D}_{\mathrm{t}}^{\alpha}(\mathrm{u}(\xi))\right|\right] d \xi \\
& +\frac{\alpha-1}{\mathbb{M}(\alpha-1) \Gamma(\alpha)} \int_{0}^{\mathrm{t}}\left[\mathbf{A}_{\mathrm{g}}+\mathbf{B}_{\mathrm{g}}|\mathrm{u}(\xi)|+\mathbf{C}_{\mathrm{g}}\left|{ }_{0}^{\mathcal{A B C}} \mathcal{D}_{\xi}^{\alpha}(\mathrm{u}(\xi))\right|\right](\mathrm{t}-\xi)^{\alpha-1} d \xi .
\end{aligned}
$$

Now

$$
\begin{aligned}
\left|{ }_{0}^{\mathcal{A B C}} \mathcal{D}_{\mathrm{t}}^{\alpha}(\mathrm{u}(\mathrm{t}))\right| & =\left|\mathrm{g}\left(\mathrm{t}, \mathrm{u}(\mathrm{t}),{ }_{0}^{\mathcal{A B C}} \mathcal{D}_{\mathrm{t}}^{\alpha} \mathrm{u}(\mathrm{t})\right)\right| \\
& \leq \mathbf{A}_{\mathrm{g}}+\mathbf{B}_{\mathrm{g}}|\mathrm{u}(\mathrm{t})|+\mathbf{C}_{\mathrm{g}}\left|{ }_{0}^{\mathcal{A B C}} \mathcal{D}_{\mathrm{t}}^{\alpha}(\mathrm{u}(\mathrm{t}))\right| .
\end{aligned}
$$

This implies

$$
\left|{ }_{0}^{\mathcal{A B C}} \mathcal{D}_{\mathrm{t}}^{\alpha}(\mathrm{u}(\mathrm{t}))\right| \leq \frac{\mathbf{A}_{\mathrm{g}}}{1-\mathbf{C}_{\mathrm{g}}}+\frac{\mathbf{B}_{\mathrm{g}}}{1-\mathbf{C}_{\mathrm{g}}}|\mathrm{u}(\mathrm{t})|
$$


Using equation (15) in equation (14), we get

$$
\|\mathcal{N} \mathrm{u}\| \leq \frac{\mathrm{b} \Gamma(\alpha+1)+\mathrm{b}^{\alpha}}{\mathbb{M}(\alpha-1) \Gamma(\alpha+1)}\left[\mathbf{A}_{\mathrm{g}}+\frac{\mathbf{A}_{\mathrm{g}} \cdot \mathbf{C}_{\mathrm{g}}}{1-\mathbf{C}_{\mathrm{g}}}+\left(\mathbf{B}_{\mathrm{g}}+\frac{\mathbf{B}_{\mathrm{g}}}{1-\mathbf{C}_{\mathrm{g}}}\right) \mathfrak{M}\right]
$$

Hence, $\mathcal{N}$ is bounded. Now to prove equicontinuity for $\mathcal{N}$, let $t_{1}<t_{2} \in \mathcal{J}$, then

$$
\begin{aligned}
\left|\mathcal{N u}\left(\mathrm{t}_{2}\right)-\mathcal{N} \mathrm{u}\left(\mathrm{t}_{1}\right)\right| & \leq \frac{2-\alpha}{\leq}\left[\int_{\mathrm{t}_{1}}^{\mathrm{t}_{2}}\left|\mathrm{~g}\left(\xi, \mathrm{u}(\xi),{ }_{0}^{\mathcal{A B C}} \mathcal{D}_{\xi}^{\alpha} \mathrm{u}(\xi)\right)\right| d \xi\right] \\
& +\frac{\alpha-1}{\mathrm{M}(\alpha-1) \Gamma(\alpha)} \int_{0}^{\mathrm{t}_{1}}\left[\left(\mathrm{t}_{1}-\xi\right)^{\alpha-1}-\left(\mathrm{t}_{2}-\xi\right)^{\alpha-1}\right]\left|\mathrm{g}\left(\xi, \mathrm{u}(\xi),{ }_{0}^{\mathcal{A B C}} \mathcal{D}_{\xi}^{\alpha} \mathrm{u}(\xi)\right)\right| d \xi \\
& +\frac{\alpha-1}{\mathbb{M}(\alpha-1) \Gamma(\alpha)} \int_{\mathrm{t}_{1}}^{\mathrm{t}_{2}}\left(\mathrm{t}_{2}-\xi\right)^{\alpha-1}\left|\mathrm{~g}\left(\xi, \mathrm{u}(\xi),_{0}^{\mathcal{A B C}} \mathcal{D}_{\xi}^{\alpha} \mathrm{u}(\xi)\right)\right| d \xi \\
\leq & \frac{2-\alpha}{\mathbb{M}(\alpha-1)} \int_{\mathrm{t}_{1}}^{\mathrm{t}_{2}}\left[\mathbf{A}_{\mathrm{g}}+\mathbf{B}_{\mathrm{g}}|\mathrm{u}(\xi)|+\mathbf{C}_{\mathrm{g}}||_{0}^{\mathcal{A B C}} \mathcal{D}_{\xi}^{\alpha} \mathrm{u}(\xi) \mid\right] d \xi \\
& +\frac{\alpha-1}{\mathbb{M}(\alpha-1) \Gamma(\alpha)} \int_{0}^{\mathrm{t}_{1}}\left[\left(\mathrm{t}_{1}-\xi\right)^{\alpha-1}-\left(\mathrm{t}_{2}-\xi\right)^{\alpha-1}\right] \\
& \times\left[\mathbf{A}_{\mathrm{g}}+\mathbf{B}_{\mathrm{g}}|\mathrm{u}(\xi)|+\left.\mathbf{C}_{\mathrm{g}}\right|_{0} ^{\mathcal{A B C}} \mathcal{D}_{\xi}^{\alpha} \mathrm{u}(\xi) \mid\right] d \xi \\
& +\frac{\alpha-1}{\mathbb{M}(\alpha-1) \Gamma(\alpha)} \int_{\mathrm{t}_{1}}^{\mathrm{t}_{2}}\left(\mathrm{t}_{2}-\xi\right)^{\alpha-1}\left[\mathbf{A}_{\mathrm{g}}+\mathbf{B}_{\mathrm{g}}|\mathrm{u}(\xi)|+\mathbf{C}_{\mathrm{g}}||_{0}^{\mathcal{A B C}} \mathcal{D}_{\xi}^{\alpha} \mathrm{u}(\xi) \mid\right] d \xi .
\end{aligned}
$$

Using relation (15) in (16), we get

$$
\begin{aligned}
& \left|\mathcal{N} \mathrm{u}\left(\mathrm{t}_{2}\right)-\mathcal{N} \mathrm{u}\left(\mathrm{t}_{1}\right)\right| \\
& \leq \frac{(2-\alpha)\left(\mathrm{t}_{2}-\mathrm{t}_{1}\right)}{\mathbb{M}(\alpha-1)}\left[\mathbf{A}_{\mathrm{g}}+\frac{\mathbf{A}_{\mathrm{g}} \cdot \mathbf{C}_{\mathrm{g}}}{1-\mathbf{C}_{\mathrm{g}}}+\left(\mathbf{B}_{\mathrm{g}}+\frac{\mathbf{B}_{\mathrm{g}}}{1-\mathbf{C}_{\mathrm{g}}}\right) \mathfrak{M}\right] \\
& \quad+\frac{(\alpha-1)\left(\mathrm{t}_{1}^{\alpha}-\mathrm{t}_{2}^{\alpha}+2\left(\mathrm{t}_{1}-\mathrm{t}_{2}\right)^{\alpha}\right)}{\mathbb{M}(\alpha-1) \Gamma(\alpha+1)}\left[\mathbf{A}_{\mathrm{g}}+\frac{\mathbf{A}_{\mathrm{g}} \cdot \mathbf{C}_{\mathrm{g}}}{1-\mathbf{C}_{\mathrm{g}}}+\left(\mathbf{B}_{\mathrm{g}}+\frac{\mathbf{B}_{\mathrm{g}}}{1-\mathbf{C}_{\mathrm{g}}}\right) \mathfrak{M}\right]
\end{aligned}
$$

This shows that $\left\|\mathcal{N} \mathrm{u}\left(\mathrm{t}_{2}\right)-\mathcal{N} \mathrm{u}\left(\mathrm{t}_{1}\right)\right\| \longrightarrow 0$ as $\mathrm{t}_{2} \longrightarrow \mathrm{t}_{1}$.

Hence, by Arzelá-Ascoli theorem, $\mathcal{N}$ is completely continuous. With all the above, the conditions of Krasnoselskii's fixed point theorem are fulfilled, and thus the BVP has at least one solution in $\mathcal{W}$.

\section{Stability results}

Stability analysis has an important impact on the theory of fractional differential equations. In fractional calculus different types of stabilities have been introduced. For our implicit BVP, we use Hyers-Ulam stabilities. In particular, we work on Hyers-Ulam, generalized Hyers-Ulam, Hyers-Ulam-Rassias, and generalized Hyers-Ulam-Rassias stability analysis. For further explanation of these definitions, one can refer to [31-34].

Definition 4 (Hyers-Ulam stable) The implicit BVP (3) is Hyers-Ulam stable if there exists a real number $\mathcal{C}_{\mathrm{g}}>0$ such that for $\epsilon>0$ and for any solution $\mathrm{u} \in \mathbb{X}$ of the inequality

$$
\left|{ }_{0}^{\mathcal{A B C}} \mathcal{D}_{\mathrm{t}}^{\alpha} \mathrm{u}(\mathrm{t})-\mathrm{g}\left(\mathrm{t}, \mathrm{u}(\mathrm{t}),{ }_{0}^{\mathcal{A B C}} \mathcal{D}_{\mathrm{t}}^{\alpha} \mathrm{u}(\mathrm{t})\right)\right| \leq \epsilon,
$$


there is the unique solution $\mathrm{u}^{\star} \in \mathbb{X}$ for our problem (3) such that

$$
\left\|\mathrm{u}-\mathrm{u}^{\star}\right\| \leq \mathcal{C}_{\mathrm{g}} \epsilon .
$$

Definition 5 (Generalized Hyers-Ulam stable) Our BVP is generalized Hyers-Ulam stable if there exists $\varphi \in \mathcal{C}[0,1], \varphi(0)=0$ such that, for any solution $\mathrm{u} \in \mathbb{X}$ of inequality (17), there is the unique solution $u^{\star} \in \mathbb{X}$ of problem (3) such that

$$
\left\|\mathrm{u}-\mathrm{u}^{\star}\right\| \leq \mathcal{C}_{\mathrm{g}} \varphi(\epsilon)
$$

Remark 1 A function $\mathrm{u} \in \mathbb{X}$ is the solution of inequality (17) if there is a function $\Psi \in$ $\mathcal{C}[0,1]$ that depends on $\mathrm{u}$ such that

(1) $|\Psi(\mathrm{t})| \leq \epsilon$;

(2) ${ }_{0}^{\mathcal{A B C}} \mathcal{D}_{\mathrm{t}}^{\alpha} \mathrm{u}(\mathrm{t})=\mathrm{g}\left(\mathrm{t}, \mathrm{u}(\mathrm{t}),{ }_{0}^{\mathcal{A} B \mathcal{B C}} \mathcal{D}_{\mathrm{t}}^{\alpha} \mathrm{u}(\mathrm{t})\right)+\Psi(\mathrm{t})$.

Definition 6 (Hyers-Ulam-Rassias stable) Our BVP is Hyers-Ulam-Rassias stable with respect to $\psi \in \mathbb{X}$ if there exists a real number $\mathcal{C}_{\mathrm{g}}>0$ such that, for $\epsilon>0$ and for any solution $\mathrm{u} \in \mathbb{X}$ of the inequality

$$
\left|{ }_{0}^{\mathcal{A B C}} \mathcal{D}_{\mathrm{t}}^{\alpha} \mathrm{u}(\mathrm{t})-\mathrm{g}\left(\mathrm{t}, \mathrm{u}(\mathrm{t}),{ }_{0}^{\mathcal{A B C}} \mathcal{D}_{\mathrm{t}}^{\alpha} \mathrm{u}(\mathrm{t})\right)\right| \leq \psi(\mathrm{t}) \epsilon,
$$

there exists the unique solution $u^{\star} \in \mathbb{X}$ for our BVP (3) such that

$$
\left\|\mathrm{u}-\mathrm{u}^{\star}\right\| \leq \mathcal{C}_{\mathrm{g}} \psi(\mathrm{t})(\epsilon)
$$

Definition 7 (Generalized Hyers-Ulam-Rassias stable) Our BVP (3) is generalized Hyers-Ulam-Rassias stable with respect to $\psi \in \mathcal{C}[0,1]$, if there is $\mathcal{C}_{\mathrm{g}}>0$ such that, for any solution $u \in \mathbb{X}$ of inequality (18), there exists the unique solution $u^{\star} \in \mathbb{X}$ of our BVP such that

$$
\left\|\mathrm{u}-\mathrm{u}^{\star}\right\| \leq \mathcal{C}_{\mathrm{g}} \psi(\mathrm{t})
$$

Remark 2 A function $u \in \mathbb{X}$ is the solution of our inequality (18) if there is a function $\Psi \in \mathcal{C}[0,1]$ that depends on u such that

$$
\begin{aligned}
& \text { (1) }|\Psi(\mathrm{t})| \leq \psi(\mathrm{t}) \epsilon \\
& \text { (2) }{ }_{0}^{\mathcal{A B C}} \mathcal{D}_{\mathrm{t}}^{\alpha} \mathrm{u}(\mathrm{t})=\mathrm{g}\left(\mathrm{t}, \mathrm{u}(\mathrm{t}),{ }_{0}^{\mathcal{A B C}} \mathcal{D}_{\mathrm{t}}^{\alpha} \mathrm{u}(\mathrm{t})\right)+\Psi(\mathrm{t}) .
\end{aligned}
$$

Lemma 3 By using Remark 1, the function $\mathrm{u} \in \mathbb{X}$ corresponding to the given problem

$$
\left\{\begin{array}{l}
{ }_{0}^{\mathcal{A B C}} \mathcal{D}_{\xi}^{\alpha} \mathrm{u}(\mathrm{t})=\mathrm{g}\left(\mathrm{t}, \mathrm{u}(\mathrm{t}),{ }_{0}^{\mathcal{A} C}{ }^{\mathcal{B} C} \mathcal{D}_{\xi}^{\alpha} \mathrm{u}(\mathrm{t})\right)+\Psi(\mathrm{t}), \quad 1<\alpha \leq 2, \mathrm{t} \in \mathcal{J}, \\
\mathrm{u}(0)=\mathrm{u}_{0}, \quad \mathrm{u}(\mathrm{b})=\mathrm{u}_{1} .
\end{array}\right.
$$

satisfies the inequality

$$
|\mathrm{u}(\mathrm{t})-\mathcal{H} \mathrm{u}(\mathrm{t})| \leq \mathcal{C}_{\mathrm{g} \alpha} \epsilon, \quad \forall \mathrm{t} \in \mathcal{J} .
$$


Now, one has

$$
\begin{aligned}
\mathcal{H} \mathrm{u}(\mathrm{t})= & \frac{\mathrm{tu} \mathrm{u}_{1}+\mathrm{u}_{0}(\mathrm{~b}-\mathrm{t})}{\mathrm{b}}-\frac{\mathrm{t}(2-\alpha)}{\mathrm{bM}(\alpha-1)} \int_{0}^{\mathrm{b}} \mathrm{g}\left(\xi, \mathrm{u}(\xi),{ }_{0}^{\mathcal{A B C}} \mathcal{D}_{\xi}^{\alpha} \mathrm{u}(\xi)\right) d \xi \\
& -\frac{\mathrm{t}(\alpha-1)}{\mathrm{bM}(\alpha-1) \Gamma(\alpha)} \int_{0}^{\mathrm{b}} \mathrm{g}\left(\xi, \mathrm{u}(\xi),{ }_{0}^{\mathcal{A B C}} \mathcal{D}_{\xi}^{\alpha} \mathrm{u}(\xi)\right)(\mathrm{b}-\xi)^{\alpha-1} d \xi \\
& +\frac{2-\alpha}{\mathbb{M}(\alpha-1)} \int_{0}^{\mathrm{t}} \mathrm{g}\left(\xi, \mathrm{u}(\xi),{ }_{0}^{\mathcal{A}}{ }^{\mathcal{B C}} \mathcal{D}_{\xi}^{\alpha} \mathrm{u}(\xi)\right) d \xi \\
& +\frac{\alpha-1}{\mathbb{M}(\alpha-1) \Gamma(\alpha)} \int_{0}^{\mathrm{t}} \mathrm{g}\left(\xi, \mathrm{u}(\xi),{ }_{0}^{\mathcal{A B C}} \mathcal{D}_{\xi}^{\alpha} \mathrm{u}(\xi)\right)(\mathrm{t}-\xi)^{\alpha-1} d \xi
\end{aligned}
$$

and we have

$$
\mathcal{C}_{\mathrm{g}, \alpha}=\frac{4\left(\mathrm{~b} \Gamma(\alpha+1)+\mathrm{b}^{\alpha}\right)}{\mathrm{M}(\alpha-1) \Gamma(\alpha+1)}
$$

Proof In view of Lemma 2 and equation (19), we get

$$
\begin{aligned}
\mathrm{u}(\mathrm{t})= & \mathfrak{c}_{0}+\mathfrak{c}_{1} \mathrm{t}+\frac{2-\alpha}{\mathrm{M}(\alpha-1)} \int_{0}^{\mathrm{t}}\left(\mathrm{g}\left(\xi, \mathrm{u}(\xi),{ }_{0}^{\mathcal{A B C}} \mathcal{D}_{\xi}^{\alpha} \mathrm{u}(\xi)\right)+\Psi(\mathrm{t})\right) d \xi \\
& +\frac{\alpha-1}{\mathbb{M}(\alpha-1) \Gamma(\alpha)} \int_{0}^{\mathrm{t}}\left(\mathrm{g}\left(\xi, \mathrm{u}(\xi),{ }_{0}^{\mathcal{A B C}} \mathcal{D}_{\xi}^{\alpha} \mathrm{u}(\xi)\right)+\Psi(\mathrm{t})\right)(\mathrm{t}-\xi)^{\alpha-1} d \xi \\
\mathrm{u}(\mathrm{t})= & \frac{\mathrm{tu}_{1}+\mathrm{u}_{0}(\mathrm{~b}-\mathrm{t})}{\mathrm{b}}-\frac{\mathrm{t}(2-\alpha)}{\mathrm{bM}(\alpha-1)} \int_{0}^{\mathrm{b}} \mathrm{g}\left(\xi, \mathrm{u}(\xi),{ }_{0}^{\mathcal{A B C}} \mathcal{D}_{\xi}^{\alpha} \mathrm{u}(\xi)\right) d \xi \\
& -\frac{\mathrm{t}(\alpha-1)}{\mathrm{bM}(\alpha-1) \Gamma(\alpha)} \int_{0}^{\mathrm{b}} \mathrm{g}\left(\xi, \mathrm{u}(\xi),{ }_{0}^{\mathcal{A B C}} \mathcal{D}_{\xi}^{\alpha} \mathrm{u}(\xi)\right)(\mathrm{b}-\xi)^{\alpha-1} d \xi \\
& +\frac{2-\alpha}{\mathbb{M}(\alpha-1)} \int_{0}^{\mathrm{t}} \mathrm{g}\left(\xi, \mathrm{u}(\xi),{ }_{0}^{\mathcal{A B C}} \mathcal{D}_{\xi}^{\alpha} \mathrm{u}(\xi)\right) d \xi \\
& +\frac{\alpha-1}{\mathbb{M}(\alpha-1) \Gamma(\alpha)} \int_{0}^{\mathrm{t}} \mathrm{g}\left(\xi, \mathrm{u}(\xi),{ }_{0}^{\mathcal{A B C}} \mathcal{D}_{\xi}^{\alpha} \mathrm{u}(\xi)\right)(\mathrm{t}-\xi)^{\alpha-1} d \xi \\
& -\frac{\mathrm{t}(2-\alpha)}{\mathrm{bM}(\alpha-1)} \int_{0}^{\mathrm{b}} \Psi(\xi) d \xi-\frac{\mathrm{t}(\alpha-1)}{\mathrm{bM}(\alpha-1) \Gamma(\alpha)} \int_{0}^{\mathrm{b}} \Psi(\xi)(\mathrm{b}-\xi)^{\alpha-1} d \xi \\
& +\frac{2-\alpha}{\mathbb{M}(\alpha-1)} \int_{0}^{\mathrm{t}} \Psi(\xi) d \xi+\frac{\alpha-1}{\mathbb{M}(\alpha-1) \Gamma(\alpha)} \int_{0}^{\mathrm{t}} \Psi(\xi)(\mathrm{t}-\xi)^{\alpha-1} d \xi .
\end{aligned}
$$

Now from the above equation we conclude our result as follows:

$$
|\mathrm{u}(\mathrm{t})-\mathcal{H} \mathrm{u}(\mathrm{t})| \leq \mathcal{C}_{\mathrm{g}, \alpha} \epsilon
$$

Theorem 4 In view of assumption $\left(\mathrm{A}_{1}\right)$ and Lemma 3, the solution of our boundary value problem is Hyers-Ulam stable and generalized Hyers-Ulam stable if $4 \mathrm{~g}_{\mathrm{g}} \mathcal{C}_{\mathrm{g}, \alpha}<1$.

Proof If $\mathrm{u}^{\star}(\mathrm{t})$ is the unique solution and $\mathrm{u}(\mathrm{t})$ is any solution of our modeled problem, then we have

$$
\left|\mathrm{u}(\mathrm{t})-\mathrm{u}^{\star}(\mathrm{t})\right|=\left|\mathrm{u}(\mathrm{t})-\mathcal{H} \mathrm{u}^{\star}(\mathrm{t})\right|
$$




$$
\begin{aligned}
& =\left|\mathrm{u}(\mathrm{t})-\mathcal{H} \mathrm{u}(\mathrm{t})+\mathcal{H} \mathrm{u}(\mathrm{t})-\mathcal{H} \mathrm{u}^{\star}(\mathrm{t})\right| \\
& \leq|\mathrm{u}(\mathrm{t})-\mathcal{H} \mathrm{u}(\mathrm{t})|+\left|\mathcal{H} \mathrm{u}(\mathrm{t})-\mathcal{H} \mathrm{u}^{\star}(\mathrm{t})\right| \\
& \leq \mathcal{C}_{\mathrm{g}, \alpha} \epsilon+|\mathcal{H} \mathrm{u}(\mathrm{t})-\mathcal{H} \mathrm{u}(\mathrm{t})| \\
& \leq \mathcal{C}_{\mathrm{g}, \alpha} \epsilon+4\left(\mathcal{L}_{\mathrm{g}}+\frac{\mathcal{L}_{\mathrm{g}}^{2}}{1-\mathcal{L}_{\mathrm{g}}}\right) \frac{\mathrm{b} \Gamma(\alpha+1)+\mathrm{b}^{\alpha}}{\mathrm{M}(\alpha-1) \Gamma(\alpha+1)}\left\|\mathrm{u}-\mathrm{u}^{\star}\right\| \\
& \leq \mathcal{C}_{\mathrm{g}, \alpha} \epsilon+4 \mathrm{~g}_{\mathrm{g}} \mathcal{C}_{\mathrm{g}, \alpha}\left\|\mathrm{u}-\mathrm{u}^{\star}\right\|,
\end{aligned}
$$

where

$$
\mathrm{g}_{\mathrm{g}}=\left(\mathcal{L}_{\mathrm{g}}+\frac{\mathcal{L}_{\mathrm{g}}^{2}}{1-\mathcal{L}_{\mathrm{g}}}\right)
$$

From above we have

$$
\left\|\mathrm{u}-\mathrm{u}^{\star}\right\| \leq \frac{\mathcal{C}_{\mathrm{g}, \alpha} \epsilon}{1-4 \mathrm{~g}_{\mathrm{g}} \mathcal{C}_{\mathrm{g}, \alpha}} .
$$

Let

$$
\begin{gathered}
\mathfrak{E}_{\mathrm{g}}=\frac{\mathcal{C}_{\mathrm{g}, \alpha}}{1-4 \mathrm{~g}_{\mathrm{g}} \mathcal{C}_{\mathrm{g}, \alpha}} \\
\left\|\mathrm{u}-\mathrm{u}^{\star}\right\| \leq \mathfrak{E}_{\mathrm{g}} \epsilon .
\end{gathered}
$$

Thus our solution is Hyers-Ulam stable. And if $\psi(\epsilon)=\epsilon$, thus our problem is also generalized Hyers-Ulam stable.

Lemma 4 In view of Remark 2, the BVP mentioned in Lemma 3 satisfies the inequality

$$
\left|\mathrm{u}(\mathrm{t})-\mathcal{H}\left(\mathrm{t}, \mathrm{u}(\mathrm{t}),{ }_{0}^{\mathcal{A B C}} \mathcal{D}_{\mathrm{t}}^{\alpha} \mathrm{u}(\mathrm{t})\right)\right| \leq \mathcal{C}_{\mathrm{g}, \alpha} \in \Psi(\mathrm{t}) \quad \forall \mathrm{t} \in[0, \mathrm{~b}] .
$$

Proof We prove this inequality in view of Remark 2, calculations are the same as in Lemma 3.

Theorem 5 In view of Lemma 4 and under consideration of assumption $\mathrm{A}_{1}$, the solution of our problem is Hyers-Ulam-Rassias stable and generalized Hyers-Ulam-Rassias stable if $4 \mathrm{~g}_{\mathrm{g}} \mathcal{C}_{\mathrm{g}, \alpha}<1$.

Proof

$$
\begin{aligned}
\left|\mathrm{u}(\mathrm{t})-\mathrm{u}^{\star}(\mathrm{t})\right| & =\left|\mathrm{u}(\mathrm{t})-\mathcal{H} \mathrm{u}^{\star}(\mathrm{t})\right| \\
& =\left|\mathrm{u}(\mathrm{t})-\mathcal{H} \mathrm{u}(\mathrm{t})+\mathcal{H} \mathrm{u}(\mathrm{t})-\mathcal{H} \mathrm{u}^{\star}(\mathrm{t})\right| \\
& \leq|\mathrm{u}(\mathrm{t})-\mathcal{H} \mathrm{u}(\mathrm{t})|+\left|\mathcal{H} \mathrm{u}(\mathrm{t})-\mathcal{H} \mathrm{u}^{\star}(\mathrm{t})\right| \\
& \leq \mathcal{C}_{\mathrm{g}, \alpha} \in \psi(\mathrm{t})+\left|\mathcal{H} \mathrm{u}(\mathrm{t})-\mathcal{H} \mathrm{u}^{\star}(\mathrm{t})\right| \\
& \leq \mathcal{C}_{\mathrm{g}, \alpha} \in \psi(\mathrm{t})+4 \mathrm{~g}_{\mathrm{g}} \mathcal{C}_{\mathrm{g}, \alpha}\left\|\mathrm{u}-\mathrm{u}^{\star}\right\| .
\end{aligned}
$$


After simplification, from (22), one has

$$
\left\|\mathrm{u}-\mathrm{u}^{\star}\right\| \leq \frac{\mathcal{C}_{\mathrm{g}, \alpha}}{1-4 \mathrm{~g}_{\mathrm{g}} \mathcal{C}_{\mathrm{g}, \alpha}} \epsilon \psi(\mathrm{t})
$$

Thus our problem is generalized Hyers-Ulam-Rassias stable.

\section{Illustrative examples}

In this section, we verify our boundary value problem with different examples.

Example 1 Considered the following BVP:

$$
\left\{\begin{array}{l}
\mathcal{A B C}^{\mathcal{B} C} \mathcal{D}_{\mathrm{t}}^{\frac{3}{2}} \mathrm{u}(\mathrm{t})=\frac{\mathrm{t} \ln \mathrm{t}}{20}+\frac{\mathrm{u}(\mathrm{t}) e^{-\sin \mathrm{t}}}{90}+\frac{\mathcal{A B C}^{\mathcal{B} C} \mathcal{D}_{\mathrm{t}}^{\alpha} \mathrm{u}\left(\frac{\mathrm{t}}{3}\right)}{90 e^{-\mathrm{c}-\cos \mathrm{t}}}, \quad \mathrm{t} \in[0,1] \\
\mathrm{u}(0)=0, \quad \mathrm{u}(1)=0 .
\end{array}\right.
$$

Here, $\mathrm{b}=1$ and $\alpha=\frac{3}{2}$

$$
\mathrm{g}(\mathrm{t}, \mathrm{u}, \mathrm{v})=\frac{\mathrm{t} \ln \mathrm{t}}{20}+\frac{\mathrm{u}(\mathrm{t}) e^{-\sin \mathrm{t}}}{90}+\frac{{ }^{\mathcal{A B C}} \mathcal{D}_{\mathrm{t}}^{\alpha} \mathrm{u}\left(\frac{\mathrm{t}}{3}\right)}{90 e^{-\mathrm{t}-\cos \mathrm{t}}}
$$

Let $\mathrm{u}, \overline{\mathrm{u}}, \mathrm{v}, \overline{\mathrm{v}} \in \mathbb{X}$

$$
\begin{aligned}
& |\mathrm{g}(\mathrm{t}, \mathrm{u}, \mathrm{v})-\mathrm{g}(\mathrm{t}, \overline{\mathrm{u}}, \overline{\mathrm{v}})| \\
& \quad=\left|\left[\frac{\mathrm{t} \ln \mathrm{t}}{20}+\frac{\mathrm{u}(\mathrm{t}) e^{-\sin \mathrm{t}}}{90}+\frac{{ }_{0}^{\mathcal{A B C}} \mathcal{D}_{\mathrm{t}}^{\frac{3}{2}} \mathrm{u}\left(\frac{\mathrm{t}}{3}\right)}{90 e^{-\mathrm{t}-\cos \mathrm{t}}}\right]-\left[\frac{\mathrm{t} \ln \mathrm{t}}{20}+\frac{\overline{\mathrm{u}}(\mathrm{t}) e^{-\sin \mathrm{t}}}{90}+\frac{\mathcal{A B C}_{0} \mathcal{D}_{\mathrm{t}}^{\frac{3}{2}} \overline{\mathrm{u}}\left(\frac{\mathrm{t}}{3}\right)}{90 e^{-\mathrm{t}-\cos \mathrm{t}}}\right]\right| \\
& \quad \leq \frac{1}{90}|\mathrm{u}-\overline{\mathrm{u}}|+\frac{1}{90}|\mathrm{v}-\overline{\mathrm{v}}| .
\end{aligned}
$$

Now, using assumption $\left(\mathrm{A}_{2}\right)$, we obtain

$$
\begin{aligned}
|\mathrm{g}(\mathrm{t}, \mathrm{u}, \mathrm{v})| & =\left|\frac{\mathrm{t} \ln \mathrm{t}}{20}+\frac{\mathrm{u}(\mathrm{t}) e^{-\sin \mathrm{t}}}{90}+\frac{{ }_{0}^{\mathcal{A B C}} \mathcal{D}_{\mathrm{t}}^{\frac{3}{2}} \mathrm{u}\left(\frac{\mathrm{t}}{3}\right)}{90 e^{-\mathrm{t}-\cos \mathrm{t}}}\right| \\
& \leq \frac{1}{20}+\frac{1}{90}|\mathrm{u}|+\frac{1}{90}|\mathrm{v}|
\end{aligned}
$$

Thus, we have $\mathcal{L}_{\mathrm{g}}=\frac{1}{90}, \alpha=\frac{3}{2}, \mathbf{A}_{\mathrm{g}}=\frac{1}{20}, \mathbf{B}_{\mathrm{g}}=\frac{1}{90}$, and $\mathbf{C}_{\mathrm{g}}=\frac{1}{90}$

$$
4\left(\mathcal{L}_{\mathrm{g}}+\frac{\mathcal{L}_{\mathrm{g}}^{2}}{1-\mathcal{L}_{\mathrm{g}}}\right) \frac{\mathrm{b} \Gamma(\alpha+1)+\mathrm{b}^{\alpha}}{\mathrm{M}(\alpha-1) \Gamma(\alpha+1)}=0.008440<1
$$

Thus all the conditions for Theorem 2 are satisfied. Hence, in view of equation (9), our BVP has the unique solution. Also

$$
2\left(\mathcal{L}_{\mathrm{g}}+\frac{\mathcal{L}_{\mathrm{g}}^{2}}{1-\mathcal{L}_{\mathrm{g}}}\right) \frac{\mathrm{b} \Gamma(\alpha+1)+\mathrm{b}^{\alpha}}{\mathrm{M}(\alpha-1) \Gamma(\alpha+1)}=0.0168959<1
$$

The condition for Theorem 3 is also satisfied. Moreover, we also have $\mathcal{C}_{\mathrm{g}, \alpha} \neq 1$. Hence the solution is both Hyers-Ulam and generalized Hyers-Ulam stable. In a similar way, one 
can easily verify the conditions of Hyers-Ulam-Rassias and generalized Hyers-UlamRassias stability.

Example 2 Consider the following nonhomogeneous BVP:

$$
\left\{\begin{array}{l}
{ }_{0} \mathcal{B C} \mathcal{C} \mathcal{D}_{\mathrm{t}}^{\frac{4}{3}} \mathrm{u}(\mathrm{t})=\frac{\mathrm{t}^{2}+e^{\ln \mathrm{t}+\mathrm{t}}}{25}+\frac{e^{\sin t \cos _{\mathrm{t}} \mathrm{u}(\mathrm{t})}}{60}+\frac{e^{\mathrm{t}^{2}-\cos ^{2} \mathrm{t} A \mathcal{A} \mathcal{B}} \mathcal{D}_{\mathrm{t}}^{\frac{4}{3}} \mathrm{u}(\mathrm{t})}{60}, \quad \mathrm{t} \in[0,1], \\
\mathrm{u}(0)=e^{2 \pi}, \quad \mathrm{u}(1)=\sin \mathrm{u}\left(\frac{2 \pi}{3}\right)
\end{array}\right.
$$

Here, $\mathrm{b}=1$ and $\alpha=\frac{4}{3}$

$$
\mathrm{g}(\mathrm{t}, \mathrm{u}, \mathrm{v})=\frac{\mathrm{t}^{2}+e^{\ln \mathrm{t}+\mathrm{t}}}{25}+\frac{e^{\sin \mathrm{t} \cos \mathrm{t}} \mathrm{u}(\mathrm{t})}{60}+\frac{e^{\mathrm{t}^{2}-\cos ^{2} t \mathcal{A B C}} \mathcal{D}_{\mathrm{t}}^{\frac{4}{3}} \mathrm{u}(\mathrm{t})}{60}
$$

Let $\mathrm{u}, \overline{\mathrm{u}}, \mathrm{v}, \overline{\mathrm{v}} \in \mathbb{X}$

$$
\begin{aligned}
= & |\mathrm{g}(\mathrm{t}, \mathrm{u}, \mathrm{v})-\mathrm{g}(\mathrm{t}, \overline{\mathrm{u}}, \overline{\mathrm{v}})| \\
= & \mid\left[\frac{\mathrm{t}^{2}+e^{\ln \mathrm{t}+\mathrm{t}}}{25}+\frac{e^{\sin \mathrm{t} \cos \mathrm{t}} \mathrm{u}(\mathrm{t})}{60}+\frac{e^{\mathrm{t}^{2}-\cos ^{2} \mathcal{A B C}} \mathcal{D}_{\mathrm{t}}^{\frac{4}{3}} \mathrm{u}(\mathrm{t})}{60}\right] \\
& -\left[\frac{\mathrm{t}^{2}+e^{\ln \mathrm{t}+\mathrm{t}}}{25}+\frac{e^{\sin \mathrm{cos} \mathrm{t}} \mathrm{u}(\mathrm{t})}{60}+\frac{e^{\mathrm{t}^{2}-\cos ^{2} \mathrm{~A} \mathcal{A} \mathcal{B}} \mathcal{D}_{\mathrm{t}}^{\frac{4}{3}} \mathrm{u}(\mathrm{t})}{60}\right] \mid \\
\leq & \frac{1}{60}|\mathrm{u}-\overline{\mathrm{u}}|+\frac{1}{60}|\mathrm{v}-\overline{\mathrm{v}}| .
\end{aligned}
$$

Now, one has

$$
\begin{aligned}
|\mathrm{g}(\mathrm{t}, \mathrm{u}, \mathrm{v})| & =\left|\frac{\mathrm{t}^{2}+e^{\ln \mathrm{t}+\mathrm{t}}}{25}+\frac{e^{\sin \mathrm{t} \cos \mathrm{t}} \mathrm{u}(\mathrm{t})}{60}+\frac{e^{\mathrm{t}^{2}-\cos ^{2} \mathrm{t} \mathcal{A B C}} \mathcal{D}_{\mathrm{t}}^{4} \mathrm{u}(\mathrm{t})}{60}\right| . \\
& \leq \frac{1}{25}+\frac{1}{60}|\mathrm{u}|+\frac{1}{60}|\mathrm{v}|
\end{aligned}
$$

We have

$$
\mathcal{L}_{\mathrm{g}}=\frac{1}{60}, \quad \alpha=\frac{4}{3}, \quad \mathbf{A}_{\mathrm{g}}=\frac{1}{25}, \quad \mathbf{B}_{\mathrm{g}}=\frac{1}{60} \quad \text { and } \quad \mathbf{C}_{\mathrm{g}}=\frac{1}{60} .
$$

Thus

$$
4\left(\mathcal{L}_{\mathrm{g}}+\frac{\mathcal{L}_{\mathrm{g}}^{2}}{1-\mathcal{L}_{\mathrm{g}}}\right) \frac{\mathrm{b} \Gamma(\alpha+1)+\mathrm{b}^{\alpha}}{\mathrm{M}(\alpha-1) \Gamma(\alpha+1)}<1 .
$$

Therefore, all the conditions for Theorem 2 are satisfied. Hence, in view of equation (9), the given problem has the unique solution. Also

$$
2\left(\mathcal{L}_{\mathrm{g}}+\frac{\mathcal{L}_{\mathrm{g}}^{2}}{1-\mathcal{L}_{\mathrm{g}}}\right) \frac{\mathrm{b} \Gamma(\alpha+1)+\mathrm{b}^{\alpha}}{\mathrm{M}(\alpha-1) \Gamma(\alpha+1)}<1 .
$$

The condition for Theorem 3 is also satisfied. Moreover, we also have $\mathcal{C}_{\mathrm{g}, \alpha} \neq 1$. Hence the solution is both Hyers-Ulam and generalized Hyers-Ulam stable. In a similar way one can 
easily verify the conditions of Hyers-Ulam-Rassias and generalized Hyers-Ulam-Rassias stability.

Example 3 Here we take another BVP as follows:

$$
\left\{\begin{array}{l}
{ }_{0} \mathcal{B C} C \mathcal{D}_{\mathrm{t}}^{\frac{3}{2}} \mathrm{u}(\mathrm{t})=\frac{\sin (\mathrm{t})}{\mathrm{t}+50}+\frac{e^{-\mathrm{t}} \mathrm{u}(\mathrm{t})}{100}+\frac{e^{-\mathrm{t}^{2}}{ }_{0} \mathcal{A B C} \mathcal{D}_{\mathrm{t}}^{\frac{3}{2}} \mathrm{u}(\mathrm{t})}{100}, \quad \mathrm{t} \in[0,1], \\
\mathrm{u}(0)=1, \quad \mathrm{u}(1)=2 .
\end{array}\right.
$$

One has $\mathrm{b}=1$ and $\alpha=\frac{3}{2}$

$$
\mathrm{g}(\mathrm{t}, \mathrm{u}, \mathrm{v})=\frac{\sin (\mathrm{t})}{\mathrm{t}+50}+\frac{e^{-\mathrm{t}} \mathrm{u}(\mathrm{t})}{100}+\frac{e^{-\mathrm{t}^{2}}{ }_{0}^{\mathcal{A B C}} \mathcal{D}_{\mathrm{t}}^{\frac{3}{2}} \mathrm{u}(\mathrm{t})}{100} .
$$

Let $\mathrm{u}, \overline{\mathrm{u}}, \mathrm{v}, \overline{\mathrm{v}} \in \mathbb{X}$, one has

$$
|g(t, u, v)-g(t, \bar{u}, \bar{v})| \leq \frac{1}{100}|u-\bar{u}|+\frac{1}{100}|v-\bar{v}| .
$$

Now

$$
\begin{aligned}
|\mathrm{g}(\mathrm{t}, \mathrm{u}, \mathrm{v})| & =\left|\frac{\sin (\mathrm{t})}{\mathrm{t}+50}+\frac{e^{-\mathrm{t}} \mathrm{u}(\mathrm{t})}{100}+\frac{e^{-\mathrm{t}^{2}}{ }_{0} \mathcal{B C}^{-} \mathcal{D}_{\mathrm{t}}^{\frac{3}{2}} \mathrm{u}(\mathrm{t})}{100}\right| \\
& \leq \frac{1}{50}+\frac{1}{100}|\mathrm{u}|+\frac{1}{100}|\mathrm{v}| .
\end{aligned}
$$

We have

$$
\mathcal{L}_{\mathrm{g}}=\frac{1}{100}, \quad \alpha=\frac{3}{2}, \quad \mathbf{A}_{\mathrm{g}}=\frac{1}{50}, \quad \mathbf{B}_{\mathrm{g}}=\frac{1}{100} \quad \text { and } \quad \mathbf{C}_{\mathrm{g}}=\frac{1}{100} .
$$

Therefore, we have

$$
4\left(\mathcal{L}_{\mathrm{g}}+\frac{\mathcal{L}_{\mathrm{g}}^{2}}{1-\mathcal{L}_{\mathrm{g}}}\right) \frac{\mathrm{b} \Gamma(\alpha+1)+\mathrm{b}^{\alpha}}{\mathrm{M}(\alpha-1) \Gamma(\alpha+1)}<1
$$

Hence all the conditions of Theorem 2 are satisfied. Further, the condition for uniqueness is also satisfied. Also

$$
2\left(\mathcal{L}_{\mathrm{g}}+\frac{\mathcal{L}_{\mathrm{g}}^{2}}{1-\mathcal{L}_{\mathrm{g}}}\right) \frac{\mathrm{b} \Gamma(\alpha+1)+\mathrm{b}^{\alpha}}{\mathrm{M}(\alpha-1) \Gamma(\alpha+1)}<1 .
$$

Also the conditions of Theorem 3 are satisfied. Hence, we also have $\mathcal{C}_{\mathrm{g}, \alpha} \neq 1$. Thus the solution is both Hyers-Ulam and generalized Hyers-Ulam stable. In a similar way one can easily verify the conditions of Hyers-Ulam-Rassias and generalized Hyers-UlamRassias stability.

\section{Conclusion}

We have effectively achieved several necessary conditions describing the stability and existence hypotheses for a class of BVP including $(\mathcal{A B C})$ fractional derivative and integration. Under the consideration of fixed point theorems like Banach and Krasnoselskii, the 
necessary outcomes have been set up. Moreover, by an appropriate use of concepts from nonlinear analysis, some sufficient outcomes for various types of Hyers-Ulam stability have been developed. By giving appropriate examples, all the theoretical results have been testified. In future such type of analysis can be established for more general type BVPs involving the aforementioned derivative. The manuscripts [35-39] are useful for any further investigations.

\section{Acknowledgements}

We are thankful to the reviewers for useful comments. All authors have read and approved the revised version.

\section{Funding}

The first author will financially support the paper.

\section{Availability of data and materials}

Data used in this research has been included within the paper.

\section{Competing interests}

The authors declare that they have no competing interests.

\section{Authors' contributions}

All authors equally contributed to this manuscript and approved the final version.

\section{Author details}

'Department of Mathematics, COMSATS University of Islamabad, Sahiwal Campus, Punjab, Pakistan. ${ }^{2}$ Department of Mathematics, University of Malakand, Dir(L), Khyber Pakhtunkhwa, Pakistan. ${ }^{3}$ Department of Mathematics and General Sciences, Prince Sultan University, Riyadh, Saudi Arabia. ${ }^{4}$ Department of Medical Research, China Medical University, Taichung, 40402, Taiwan. ${ }^{5}$ Department of Computer Science and Information Engineering, Asia University, Taichung, Taiwan.

\section{Publisher's Note}

Springer Nature remains neutral with regard to jurisdictional claims in published maps and institutional affiliations.

Received: 9 May 2021 Accepted: 11 August 2021 Published online: 24 August 2021

\section{References}

1. Ross, B.: The development of fractional calculus 1695-1900. Hist. Math. 4(1), 75-89 (1977)

2. Kilbas, A.A., Srivastava, H.M., Trujillo, J.J.: Theory and Applications of Fractional Differential Equations. North-Holand, Amsterdam (2006)

3. Arfan, M., Shah, K., Abdeljawad, T., Mlaiki, N., Ullah, A.: A Caputo power law model predicting the spread of the COVID-19 outbreak in Pakistan. Alex. Eng. J. 60(1), 447-456 (2021)

4. Ahmad, S., Ullah, A., Al-Mdallal, Q.M., Khan, H., Shah, K., Khan, A.: Fractional order mathematical modeling of COVID-19 transmission. Chaos Solitons Fractals 139, 110256 (2020)

5. Moulai-Khatir, A., Remili, M., Beldjerd, D.: Stability, boundedness and square integrability of solutions to certain third order neutral delay differential equations. Palest. J. Math. 9(2), 880-890 (2020)

6. Bachir, F.S., Abbas, S.A.I.D., Benbachir, M., Benchohra, M.: Hilfer-Hadamard fractional differential equations; existence and attractivity. Adv. Theory Nonlinear Anal. Appl. 5(1), 49-57 (2021)

7. Hashtaman, L.: Investigation of a class of implicit anti-periodic boundary value problems. J. Math. Anal. Model 1(2), 47-61 (2021)

8. Panda, S.K., Karapinar, E., Atangana, A.: A numerical schemes and comparisons for fixed point results with applications to the solutions of Volterra integral equations in dislocated extended b-metric space. Alex. Eng. J. 59(2), 815-827 (2020)

9. Sevinik-Adigüzel, R., Aksoy, Ü., Karapinar, E., Erhan, I.M.: Uniqueness of solution for higher-order nonlinear fractional differential equations with multi-point and integral boundary conditions. Rev. R. Acad. Cienc. Exactas Fís. Nat., Ser. A Mat. 115(3), 1-16 (2021)

10. Lazreg, J.E., Abbas, S., Benchohra, M., Karapinar, E.: Impulsive Caputo-Fabrizio fractional differential equations in b-metric spaces. Open Math. 19(1), 363-372 (2021)

11. Karapinar, E., Fulga, A., Rashid, M., Shahid, L., Aydi, H.: Large contractions on quasi-metric spaces with an application to nonlinear fractional differential equations. Mathematics 7(5), 444 (2019)

12. Atangana, A., Baleanu, D.: New fractional derivatives with nonlocal and non-singular kernel: theory and application to heat transfer model (2016)

13. Ghanbari, B., Atangana, A.: A new application of fractional Atangana-Baleanu derivatives: designing ABC-fractional masks in image processing. Phys. A, Stat. Mech. Appl. 15, 123516 (2020)

14. Baleanu, D., Jajarmi, A., Mohammadi, H., Rezapour, S.: A new study on the mathematical modelling of human liver with Caputo-Fabrizio fractional derivative. Chaos Solitons Fractals 134, 109705 (2020)

15. Tuan, N.H., Mohammadi, H., Rezapour, S.: A mathematical model for COVID-19 transmission by using the Caputo fractional derivative. Chaos Solitons Fractals 140, 110107 (2020) 
16. Mohammadi, H., Kumar, S., Rezapour, S., Etemad, S.: A theoretical study of the Caputo-Fabrizio fractional modeling for hearing loss due to Mumps virus with optimal control. Chaos Solitons Fractals 144, 110668 (2021)

17. Prasad, K.R., Leela, D., Khuddush, M.: Existence and uniqueness of positive solutions for system of $(p, q, r)$-Laplacian fractional order boundary value problems. Adv. Theory Nonlinear Anal. Appl. 5(1), 138-157 (2021)

18. Jarad, F., Abdeljawad, T., Hammouch, Z:: On a class of ordinary differential equations in the frame of Atangana-Baleanu fractional derivative. Chaos Solitons Fractals 117, 16-20 (2018)

19. Rezapour, S., Ntouyas, S.K., Iqbal, M.Q., Hussain, A., Etemad, S., Tariboon, J.: An analytical survey on the solutions of the generalized double-order-integrodifferential equation. J. Funct. Spaces 2021, Article ID 6667757 (2021)

20. Thabet, S.T., Abdo, M.S., Shah, K., Abdeljawad, T.: Study of transmission dynamics of COVID-19 mathematical model under ABC fractional order derivative. Results Phys. 19, 103507 (2020)

21. Karapinar, E., Fulga, A.: An admissible hybrid contraction with an Ulam type stability. Demonstr. Math. 52(1), 428-436 (2019)

22. Alqahtani, B., Fulga, A., Karapinar, E.: Fixed point results on $\Delta$-symmetric quasi-metric space via simulation function with an application to Ulam stability. Mathematics 6(10), 208 (2018)

23. Alsulami, H.H., Gülyaz, S., Karapinar, E., Erhan, I.M.: An Ulam stability result on quasi-b-metric-like spaces. Open Math. 14(1), 1087-1103 (2016)

24. Hassan, A.M., Karapinar, E., Alsulami, H.H.: Ulam-Hyers stability for MKC mappings via fixed point theory. J. Funct. Spaces 2016, Article ID 9623597 (2016)

25. Bota, M.F., Karapinar, E., Mlesnite, O.: Ulam-Hyers stability results for fixed point problems via-contractive mapping in (b)-metric space. Abstr. Appl. Anal. 2013, Article ID 825293 (2013)

26. Atangana, A., Baleanu, D.: New fractional derivatives with nonlocal and nonsingular kernel: theory and application to heat transfer model. Therm. Sci. 20(2), 763-769 (2016)

27. Djida, J.D., Atangana, A., Area, l.: Numerical computation of a fractional derivative with non-local and non-singular kernel. Math. Model. Nat. Phenom. 12(3), 4-13 (2017)

28. Abdeljawad, T: A Lyapunov type inequality for fractional operators with nonsingular Mittag-Leffler kernel. J. Inequal. Appl. 2017, 130 (2017)

29. Karapinar, E., Binh, H.D., Luc, N.H., Can, N.H.: On continuity of the fractional derivative of the time-fractional semilinear pseudo-parabolic systems. Adv. Differ. Equ. 2021(1), 1 (2021)

30. Burton, T.A.: A fixed-point theorem of Krasnoselskii. Appl. Math. Lett. 11(1), 85-88 (1998)

31. Ardjouni, A., Djoudi, A.: Existence and uniqueness of solutions for nonlinear implicit Caputo-Hadamard fractional differential equations with nonlocal conditions. Adv. Theory Nonlinear Anal. Appl. 3(1), 46-52 (2019)

32. Rus, I.A.: Ulam stabilities of ordinary differential equations in a Banach space. Carpath. J. Math. 26(1), 103-107 (2010)

33. Alqahtani, B., Aydi, H., Karapinar, E., Rakocevic, V.: A solution for Volterra fractional integral equations by hybrid contractions. Mathematics 7(8), 694 (2019)

34. Khan, H., Gómez-Aguilar, J.F., Khan, A., Khan, T.S.: Stability analysis for fractional order advection-reaction diffusion system. Phys. A, Stat. Mech. Appl. 521, 737-751 (2019)

35. Sevinik Adigüzel, R., Aksoy, Ü., Karapinar, E., Erhan, I.M.: On the solution of a boundary value problem associated with a fractional differential equation. Math. Methods Appl. Sci. (2020). https://doi.org/10.1002/mma.6652

36. Kilbas, A.A., Saigo, M., Saxena, R.K.: Generalized Mittag-Leffler function and generalized fractional calculus operators. Integral Transforms Spec. Funct. 15(1), 31-49 (2004)

37. Kilbas, A.A., Luchko, Y.F., Martinez, H., Trujillo, J.J.: Fractional Fourier transform in the framework of fractional calculus operators. Integral Transforms Spec. Funct. 21(10), 779-795 (2010)

38. Ren, X., Wang, G., Bai, Z., El-Deeb, A.A.: Maximum principle and its application to multi-index Hadamard fractional diffusion equation. Bound. Value Probl. 2019(1), 1 (2019)

39. Baleanu, D., Etemad, S., Rezapour, S.: A hybrid Caputo fractional modeling for thermostat with hybrid boundary value conditions. Bound. Value Probl. 2020(1), 1 (2020)

\section{Submit your manuscript to a SpringerOpen ${ }^{\circ}$ journal and benefit from:}

- Convenient online submission

- Rigorous peer review

- Open access: articles freely available online

- High visibility within the field

- Retaining the copyright to your article

Submit your next manuscript at $\gg$ springeropen.com 